\title{
Automated exploit detection using path profiling The disposition should matter, not the position
}

\author{
George Stergiopoulos ${ }^{1}$, Panagiotis Petsanas ${ }^{1}$, Panagiotis Katsaros ${ }^{2}$, Dimitris Gritzalis ${ }^{1}$ \\ ${ }^{1}$ Information Security and Critical Infrastructure Protection Laboratory \\ Dept. of Informatics, Athens University of Economics \& Business (AUEB), Greece \\ \{geostergiop,dgrit\}@aueb.gr \\ ${ }^{2}$ Dept. of Informatics, Aristotle University of Thessaloniki, Greece \\ \{katsaros\}@csd.auth.gr
}

Keywords: code exploits, software vulnerabilities, source code classification, fuzzy logic, tainted object propagation.

Abstract: $\quad$ Recent advances in static and dynamic program analysis resulted in tools capable to detect various types of security bugs in the Applications under Test (AUTs). However, any such analysis is designed for a priori specified types of bugs and it is characterized by some rate of false positives or even false negatives and certain scalability limitations. We present a new analysis and source code classification technique, and a prototype tool aiming to aid code reviews in the detection of general information flow dependent bugs. Our approach is based on classifying the criticality of likely exploits in the source code using two measuring functions, namely Severity and Vulnerability. For an AUT, we analyse every single pair of input vector and program sink in an execution path, which we call an Information Block (IB). A classification technique is introduced for quantifying the Severity (danger level) of an IB by static analysis and computation of its Entropy Loss. An IB's Vulnerability is quantified using a tainted object propagation analysis along with a Fuzzy Logic system. Possible exploits are then characterized with respect to their Risk by combining the computed Severity and Vulnerability measurements through an aggregation operation over two fuzzy sets. An IB is characterized of a high risk, when both its Severity and Vulnerability rankings have been found to be above the low zone. In this case, a detected code exploit is reported by our prototype tool, called Entroine. The effectiveness of our approach has been tested by analysing 45 Java programs of NIST's Juliet Test Suite, which implement three different common weakness exploits. All existing code exploits were detected without any false positive.

\section{INTRODUCTION}

Vulnerabilities of an Application under Test (AUT) can be detected using advanced techniques of static and dynamic analysis. These techniques have been proven effective in analysing code for a priori specified flaws (e.g. Time Of Check, Time Of Use errors, widely known as TOCTOUs), but they do not go far enough in the detection of a previously unspecified form of information flow dependent flaws. Moreover, a U.S. commerce Department agency, the National Institute of Software and Technology (NIST) published a report (Okun, 2013) which indicates that most tools still generate relatively high numbers of false negatives and false positives, whereas their analysis scalability to very big programs is questionable. There are though many tools that shine on specific types of vulnerabilities, but it is clear that there is no overall "best" tool with a high detection rate in multiple exploit categories (NSA, 2011) (Rutar, 2004).

We elaborate on an analysis approach based on the classification and the criticality assessment of the program's execution paths, with each path representing a sequence of program points from one location to another location of the program's control flow graph. Our technique has been implemented in Entroine, a prototype tool for the analysis of Java code. Entroine analyses the AUT's code for possible flaws by classifying the execution paths based on their Entropy Loss, thus producing data, which are processed by a mathematical fuzzy logic system. More precisely, Entroine processes structures called information blocks (IBs), with each of them containing information for execution paths, variables and program instructions on the paths. Only a subset of 
all possible execution paths is examined: the paths from locations associated with input vectors to locations corresponding to information flow sinks. IBs are classified in two different groups of sets as follows:

- the Severity sets that quantify the danger level for the execution paths (the impact that an exploit would have, if it would be manifested in the path);

- the Vulnerability sets that quantify detected vulnerabilities based on a variable usage analysis (tainted object propagation and validation of sanitization checks, in which the data context of variables is checked).

The method consists of the following components:

i. A static analysis based on the BCEL library (Dahm, 2003) creates the control flow graph, which is then parsed to get information about variable usages. It is thus possible to detect input data vectors, control-flow locations and instructions that enforce context checks on variable data. Entroine then maps the execution paths for the AUT's variables and, more specifically, only those locations, where the program's execution can follow different paths (execution flow branching points).

ii. A classification approach that combines output from (a) to create IBs. Each IB is classified using statistical Entropy Loss and the two fuzzy membership sets, namely Severity and Vulnerability.

iii. A Fuzzy Logic system for quantifying the overall Risk for each IB, based on linguistic variables and Severity and Vulnerability classification ratings.

The main contributions of this paper are summarized as follows:

1. We introduce a program analysis technique for our classification system. Based on the control flow graph and our Fuzzy Logic ranking system only a limited number of execution paths and statements have to be analysed.

2. We propose a Risk classification of program locations using two membership functions, one for the identified Severity (Entropy Loss) and another one for the Vulnerability level.

3. We present our prototype tool. By using the Vulnerability and Severity classifications, we realized that the number of false positives for our detection technique is lowered. In addition, Entroine warned for elevated danger levels in program locations where a false negative could have occurred.
4. We provide test results from applying Entroine to the Juliet Test Suite that has been proposed by NIST to study the effectiveness of code analysis tools (NSA, 2011). Entroine detected all common weaknesses used upon, without having reported any false positive.

In Section 2, we report recent results in related research. In Section 3, we expose the theoretical underpinnings of our method. Section 4 provides technical details for the implementation of our method in Entroine and section 5 presents our experiments and reports metrics and detection coverage in all tests.

\section{RELATED WORK}

Previously proposed analysis techniques based on tainted object propagation such as the one in (Livshits, 2005) mostly focus on how to formulate various classes of security vulnerabilities as instances of the general taint analysis problem. These approaches do not explicitly model the program's control flow and it is therefore possible to miss-flag sanitized input, thus resulting in false positives. Furthermore, there is no easy general approach to avoid the possibility of false negatives. This type of analysis does not suffer a potential state space explosion, but its scalability is directly connected to the analysis sensitivity characteristics (path and context sensitivity) and there is an inherent trade-off between the analysis scalability and the resulting precision/recall.

Regarding well-known static analysis tools, it is worth to mention FindBugs (Hovemeyer, 2014), which is used to detect more than 300 code defects that are usually classified in diverse categories, including those analysed by tainted object propagation. The principle of most of the FindBug's bug detectors is to identifying low-hanging fruits, i.e. to cheaply detect likely defects or program points where the programmer's attention should be focused (Ayewah, 2008). Other tools, such as (CodePro), (UCDetector), (Pmd) and (Coverity) are well-known for their capability to detect numerous bugs, but related research in (Tripathi, 2014) has shown that their main focus is centred around specific bug types like null pointer exceptions, explicit import-export and not those, for which a taint analysis is required (XSS, OS executions etc.). In (Tripathi, 2014), a relatively low detection rate is reported for many of the aforementioned tools for some variants of important bug types (null pointer exception, user injections and non-black final instance). To the best of our knowledge, none of the aforementioned tools implements a mechanism to cope with the possibility of false negatives. 
Pixy (Jovanovic, 2010), a prototype implementing a flow-sensitive, inter-procedural and contextsensitive dataflow, alias and literal analysis, is a new tool that further develops pre-existing analyses. It mainly aims to detect cross-site scripting vulnerabilities in PHP scripts, but a false positive rate is at around $50 \%$ (i.e., one false positive for each detected vulnerability) has been reported and no mechanism has been implemented to mitigate the problem.

Other researchers try to detect code flaws using program slicing. (Weiser, 1981) introduced a program slicing technique and applied it to debugging. The main drawback is that the slice set often contains too many program entities, which in some cases can correspond to the whole program. (Zhang, 2006) presents a technique that uses a threshold to prune the computed backward slice. A limitation is that this technique does not account for the strength of the dependences between program entities, nor the likelihood for each program entity to be a failure cause. Another limitation is that the slice sets can be sometimes very large. Finally, no information is provided by any of these techniques for how to start searching for a code flaw.

Researchers in (Doupe, 2011), focus exclusively on specific flaws found in web applications as in (Balzarotti, 2007), where various analysis techniques are combined to identify multi-module vulnerabilities. None of these techniques attempts to profile the danger level in the program's behaviour. In (Stergiopoulos, 2011) (Stergiopoulos, 2012) and (Stergiopoulos, 2014), we have presented the APP_LogGIC tool for source case analysis, but we focused on logical errors instead of program exploits. and our ranking method was not based on a statistical classification of the source code.

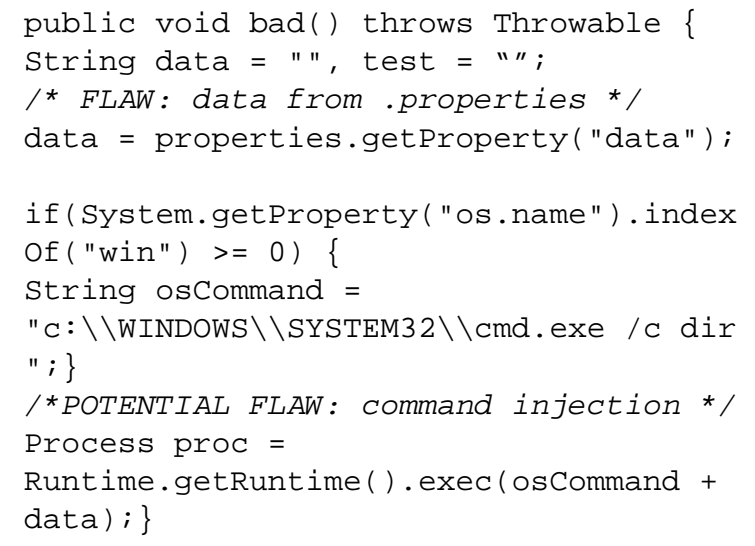

Figure 1. NIST's command injection example.

\section{METHODOLOGY}

Let us consider the example shown in Figure 1 from the Juliet Test Suite, a collection of programs for testing source code analyzers (Boland, 2012).

Example 1. Variable data in Figure 1 is assigned data originated from the source proper ties.getProperty. Then, it is used in the sink instruction getRuntime() . exec without being checked previously or having sanitized the variable's contents, as it should have happened. Our method will detect and analyze the execution path starting from the invocation of getProperty ("data") and ending with the exec()call, thus revealing the exploit present in the source code.

Definition 1. Given the set $\mathrm{T}$ of all transitions in the control flow graph of an AUT, an information block IB is a structure, containing a set of instructions I and a set of transitions $\mathrm{Ti} \subseteq \mathrm{T}$ enabled at the corresponding program points, along with information about data assignments on variables used in sets I and Ti.

Our method outputs a Risk value (ranging from one to five) that denotes the overall danger level of an IB. The Risk is quantified by means of a source code classification system using Fuzzy Logic to flag exploits (Cingolani, 2012). This classification technique aims to confront two important problems: the large data sets of the AUT analysis and the possible false positives and false negatives when trying to detect specific vulnerabilities. Regarding the first mentioned problem, the Entroine tool can help auditors to focus only to those instructions and paths that appear having a relatively high rating in its classification system. The second mentioned problem can be alleviated through Entroine's ratings that implement general criteria, which take into account the possibility of an exploit in execution paths (Vulnerability) and a path's danger level (Severity). Two measuring functions, namely Severity and Vulnerability create fuzzy sets reflecting gradually varying danger levels. Each IB gets a membership degree in these sets, which represents its danger level and it is thus classified within a broad taxonomy of exploits which is based on a wide variety of publications (Gosling, 2013)(Harold, 2006)(Mell, 2006). Membership sets act as code filters for the IBs.

Example 2. Figure 2 below depicts the Entroine's output for the program of Figure 1. Our tool detected data entry points (input vectors) and the relevant execution path, stored in the "Lines Executed" field (line numbers correspond to lines inside the source 
code Class file, depicting the execution path's instructions). Then, the IB has been classified in relevant Severity and Vulnerability ranks by analyzing checks and relations between variables. Fig. 2's Input Ranking depicts the rank assigned based on the input vector classification, in this case, the readLine ( ) instruction. Similar, Sink ranking depicts the rank assigned in the sink instruction where the exploit manifests: a rank 5 OS Injection exec() instruction.

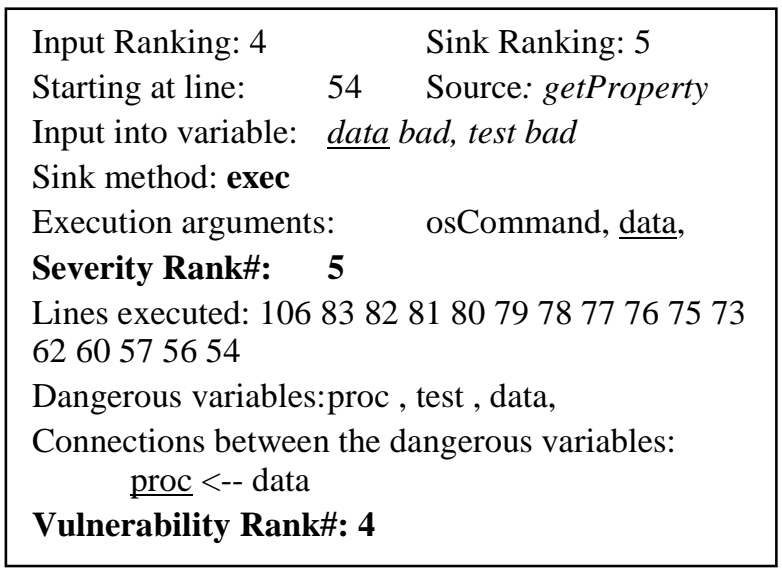

Figure 2: Information block example generated from Entroine.

In the following section, we describe in detail the way that the Severity and Vulnerability classification ranks are computed.

\subsection{Source code profiling for exploit detection}

Entroine classifies source code using two different classification mechanisms: Severity and Vulnerability. Entroine aggregates results from both to produce an distinct, overall Risk value for dangerous code points.

\subsubsection{Severity}

For an information block IB, Severity(IB) measures the membership degree of its path $\pi$ in a Severity fuzzy set. Severity(IB) reflects the relative impact on an IB's execution path $\pi$, if an exploit were to manifest on $\pi$. According to (Stoneburner, 2002) by the National Institute of Software and Technology (NIST), the impact of an exploit on a program's execution can be captured by syntactical characteristics that determine the program's execution flow, i.e. the input vectors and branch condi- tions (e.g. conditional statements). Variables used in each transition of the execution path are weighted based on how they affect the control flow. Thus, variables that directly affect the control flow or are known to manifest exploits (e.g. they input data, used in branch conditions or affect the system) are considered dangerous.

Definition 2. Given the information block IB, with a set of variables and their execution paths, we define Severity as

$$
\text { Severity }(\text { IB })=v \in[0,5]
$$

measuring the severity of IB on a Likert-type scale from 1 to 5 .

Likert scales are a convenient way to quantify facts (Albaum, 1997) that in our case refer to a program's control flow. If an exploit were to manifest on an execution path within an IB, the scale-range captures the intensity of its impact in the AUT's execution flow. Statistical Entropy Loss classifies execution paths and their information blocks in one of five Severity categories, one (1) through five (5). Categories are then grouped into Fuzzy Logic sets using labels: high Severity (4-5), medium (3) or low (1 or 2$)$.

\subsubsection{Entropy Loss as a statistical function for Severity measurement}

Evaluation of the membership degree of each execution path in the Severity set can be based on a well-defined statistical measure. To assign Severity ranks, continuous weights are estimated using Prior Entropy and Entropy Loss. Finally, a fuzzy relational classifier uses these estimations to establish correlations between Severity ranks and execution paths.

Expected Entropy Loss, which is also called Information Gain, is a statistical measure (Abramson, 1963) that has been successfully applied to the problem of feature selection for information retrieval (Etzkorn, 1997). Feature selection increases both effectiveness and efficiency, since it removes noninformative terms according to corpus statistics (Yang, 1997).

Our method is based on selected features, i.e. source code instructions, which are tied to specific types of vulnerabilities (section 4.2). For example, the exec ( ) instruction is known to be tied to OS injection vulnerabilities. Thus, Entroine uses exec ( ) as a feature to classify vulnerable IBs as a detected type of OS Injection. Expected entropy loss is computed separately for each information block. It ranks features common in both positive and negative 
variable execution paths with lower values, but ranks features higher if they are effective discriminators of an exploit (Ugurel, 2002). This technique was also used for source code classification in (Glover, 2001) and (Ugurel, 2002). Here, we use the same technique, in order to classify source code into danger levels.

In the following paragraphs, we provide a brief description of the theory (Abramson, 1963). Let C be the event that indicates whether an execution path must be considered dangerous, depending on the path's transitions, and let $f$ be the event that the path does indeed contain a specific feature $f$ (e.g. the exec() instruction). Let $\bar{C}$ and $\bar{f}$ be their negations and $\operatorname{Pr}()$ their probability (computed as in section 4.3.1). The prior entropy is the probability distribution that expresses how certain we are that an execution path belongs to a specific category, before feature $\mathrm{f}$ is taken into account:

$$
e=-\operatorname{Pr}(C) \lg \operatorname{Pr}(C)-\operatorname{Pr}(\bar{C}) \lg \operatorname{Pr}(\bar{C})
$$

where $l g$ is the binary logarithm (logarithm to the base 2). The posterior entropy, when feature $\mathrm{f}$ has been detected in the path is

$$
\begin{aligned}
e_{f}=-\operatorname{Pr}(\mathrm{C} \mid f) & \lg \operatorname{Pr}(\mathrm{C} \mid f) \\
& -\operatorname{Pr}(\bar{C} \mid f) \lg \operatorname{Pr}(\bar{C} \mid f)
\end{aligned}
$$

whereas the posterior entropy, when the feature is absent is

$$
\begin{aligned}
e_{\bar{f}}=-\operatorname{Pr}(\mathrm{C} \mid \bar{f}) & \lg \operatorname{Pr}(\mathrm{C} \mid \bar{f}) \\
& -\operatorname{Pr}(\bar{C} \mid \bar{f}) \lg \operatorname{Pr}(\bar{C} \mid \bar{f})
\end{aligned}
$$

Thus the expected overall posterior entropy is given by

$$
e_{f} \operatorname{Pr}(f)+e_{\bar{f}} \operatorname{Pr}(\bar{f})
$$

and the expected entropy loss for a given feature $f$ is

$$
e-e_{f} \operatorname{Pr}(f)-e_{\bar{f}} \operatorname{Pr}(\bar{f})
$$

The expected entropy loss is always non-negative and higher scores indicate more discriminatory features.

Example 3. Let us compute the expected Entropy Loss used for the Severity classification of the program in Example 1. Our Severity function will classify the path's features (input vectors, sinks, branch statements like exec() and getProperty()) according to a taxonomy of features (Section 4.2). Five probabilities $\operatorname{Pr}(\mathrm{C})$ were computed, one for each of the five Severity ranks and the IB was classified at the Severity rank four (4) (maximum of the five $\operatorname{Pr}(\mathrm{C}) \mathrm{s}$ ). The IB's prior entropy e was then calculated for the same ranks. Prior entropy represents the current classification certainty of Entroine, i.e. the level of confidence that it has assigned the correct Severity rank. Finally, the entropy loss (in- formation gain) was calculated for each one of the detected input vectors and sinks in the execution path, for the variable data. We are interested in the highest and the lowest observed Entropy Loss (Information Gain) values:

1. The higher value of information gain is observed, the more the uncertainty for a dangerous security characteristic is lowered and the classification to a specific Rank category is therefore more robust. Also, a relatively high information gain coupled with a high probability $\operatorname{Pr}(\mathrm{C} \mid \mathrm{f})$ for sanitization provides information about features within paths that lower the Vulnerability level.

2. The lowest value of information gain (highest entropy) provides information on the most widespread and distributed security methods by showing the level of danger diffused in the AUT's execution paths.

Figure 3 below depicts the Entropy Loss output for the example path. The conclusion drawn from this output is the following:

- The highest entropy loss (information gain) is detected in method getProperty. This shows that getProperty is a defining characteristic for this Rank 5 exploit.

- The lowest entropy loss in method exec( ) also has highest probability of appearance, $\operatorname{Pr}(\mathrm{C})=1$, which basically means that exec ( ) is being used in all execution paths analyzed as potentially dangerous. This elevates the Severity level of the detected exploit significantly, because exec ( ) is prone to OS injection.

\begin{tabular}{|ll|}
\hline Prior path Entropy $=$ & $\mathbf{0 . 8 1 1 2 7 8 1 2}$ \\
Source code Severity rank: & 5 \\
Calculation exec. time: & 2 seconds \\
Entropy Loss for getInputStream & - Rank 5: \\
& 0.31127812 \\
& \\
Entropy Loss for getProperty - Rank 5: & $\mathbf{0 . 8 1 1 2 7 8 1 2}$ \\
Entropy Loss for exec in Rank 5: & 0.0 \\
& \\
\hline
\end{tabular}

Figure 3: OS injection - Entropy for Rank 5 category.

\subsubsection{Vulnerability}

Vulnerability sets define categories based on the type of detection and the method's propagation rules and each category reveals the extent to which variable values are sanitized by conditional checks (Stoneburner, 2002). As a measuring function, Vulnerability assigns IBs into vulnerability sets thus 
quantifying how certain the tool is about an exploit manifesting in a specific variable usage.

Definition 3. Given the information block IB, with a set of variables and their execution paths, we define Severity as

$$
\text { Vulnerability }(\text { IB })=v \in[\odot, 5]
$$

Ratings here also use a Likert scale (Albaum, 1997) from 1 to 5. Similarly to the Severity (IB) function, our fuzzy logic system classifies IBs in similar categories: "high” Vulnerability, “medium” or "low".

\subsubsection{Vulnerability function - Object Propa- gation and Control Flow heuristics}

Our control flow based heuristics for assigning Vulnerability ratings to information blocks are complemented by a tainted object propagation analysis. Tainted object propagation can reveal various types of attacks that are possible due to user input that has not been (properly) validated (Livshits, 2005). Variables holding input data (sources) are considered tainted. If a tainted object (or any other object derived from it) is passed as a parameter to an exploitable instruction (a sink) like the instructions executing OS commands we have a vulnerability case (Livshits, 2005).

Variables and checks enforced upon them are analysed for the following correctness criterion: all input data should be sanitized before their use (Stoneburner, 2002). Appropriate checks show: (i) whether a tainted variable is used in sinks without having previously checked its values, (ii) if data from a tainted variable is passed along and (iii) if there are instances of the input that have never been sanitized in any way.

Entroine checks tainted variable usage by analysing its corresponding execution paths and conditions enforced on them (if any) for data propagation. The tool uses explicit taint object propagation rules for the most common Java methods, such as Sys tem. exec ( ). These rules are outlined in Section 4.3.2, where the technical implementation details are discussed.

Example 4. Again, as an example, we will show how our method analyzes the program of Figure 1 to decide in which Vulnerability rank to classify the IB of Example 2. Our tainted object analysis detects that (i) an input vector assigns data to variable data and, then, (ii) data is never checked by a conditional statement like an if-statement or any other instruction known to sanitize variable data. Then (iii) variable data is used in a sink $(\operatorname{exec}())$ without further sanitization of its contents. Thus, our method will not detect any transition that lowers the Vulnerability level of the execution path in Figure 1 and will therefore assign a high rating (4) on the Vulnerability scale for the IB containing this variable-execution path pair.

Using Severity and Vulnerability thresholds, Entroine can focus only on a subset of paths for exploit detection, thus limiting the number of paths needed to traverse during its tainted propagation analysis. The execution path set is pruned twice: (i) once based on Severity measurements and the type of instruction used ("safe" paths are discarded) and (ii) again when possible exploits have been detected by using a Vulnerability rank as threshold.

\subsection{Risk}

According to OWASP, the standard risk formulation is an operation over the likelihood and the impact of a finding (OWASP):

$$
\text { Risk }=\text { Likelihood } * \text { Impact }
$$

We adopt this notion of risk into our framework for exploit detection. In our approach, for each IB an estimate of the associated risk can be computed by combining Severity(IB) and Vulnerability( IB) into a single value called Risk. We opt for an aggregation function that allows taking into account membership degrees in a Fuzzy Logic system (Cingolani, 2012):

Definition 3. Given an AUT and an information block IB with specific input vectors, corresponding variables and their execution paths $\pi \in \mathrm{P}$, function Risk(IB) is the aggregation

$$
\begin{aligned}
& \operatorname{Risk}(I B)=\operatorname{aggreg}(\text { Severity }(\text { IB }), \\
& \text { Vulnerability (IB)) }
\end{aligned}
$$

with a fuzzy set valuation

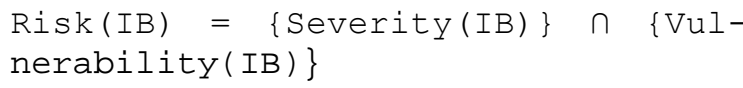

Aggregation operations on fuzzy sets are operations by which several fuzzy sets are combined to produce a single fuzzy set. Entroine applies defuzzification (Leekwijck, 1999) on the resulting set, using the Center of Gravity technique. Defuzzification is the computation of a single value from two given fuzzy sets and their corresponding membership degrees, i.e. the involvedness of each fuzzy set presented in Likert values. 
Risk ratings have the following interpretation: for two information blocks IB1 and IB2, if Risk(IB1) > Risk(IB2), then IB1 is more dangerous than IB2, in terms of how respective paths $\pi 1$ and $\pi 2$ affect the execution of the AUT and if the variable analysis detects possible exploits. In the next section, we provide technical details for the techniques used to implement the discussed analysis.

The risk of each information block is plotted separately, producing a numerical and a fuzzy result. It is calculated by the Center of Gravity technique (Leekwijck, 1999) via its Severity and Criticality assigned values. Aggregating both membership sets, produces a new membership set and, by taking the "center" (sort of an "average"), Entroine produces a discrete, numerical output.

\section{DESIGN AND IMPLEMENTA- TION}

In this section, the technical details on how Entroine was developed will be presented. The tools architecture, workflow along with technical details on how Severity and Vulnerability are calculated.

\subsection{Entroine's architecture and work- flow}

Entroine consists of three main components: a static source code analyzer (depicted with the colours orange and green in Figure 4), an Information Block constructor and, finally, the fuzzy logic system to compute the Risk (grey and yellow colours).

Static Analysis: Static code analysis uses the Java compiler to create Abstract Syntax Trees for the AUT. It provides information concerning every single method invocation, branch statements and variable assignments or declarations found in the AUT. Compiler methods (visitIf(), visit MethodInvocation ( ) etc.) were overridden, in order to analyze branch conditions and sanitization checks of variables. The following sample output shows the AST meta-data gathered for variable sig_3 in a class named Sub-system114:

DECLARE : : 12: :double: :sig_3: : $0:$ : Main22: : Subsystem114 . java

The ByteCode Engineering Library (Apache BCEL) (Dahm, 2003) is used to build the AUT's control flow graph and to extract the program's execution paths. BCEL is a library that analyzes, creates, and manipulates class files.

Information Block Creator: This component combines information obtained from the static analysis to create IBs that contain pairs of execution path input vector. Information blocks are then assigned Severity and Vulnerability ranks.

Fuzzy Logic system: The Fuzzy Logic system is implemented using the jFuzzyLogic library (Cingolani, 2012). We use it to aggregate Severity and Vulnerability sets to quantify the danger level for each IB. This aggregation classifies each IB to an overall Risk rank.

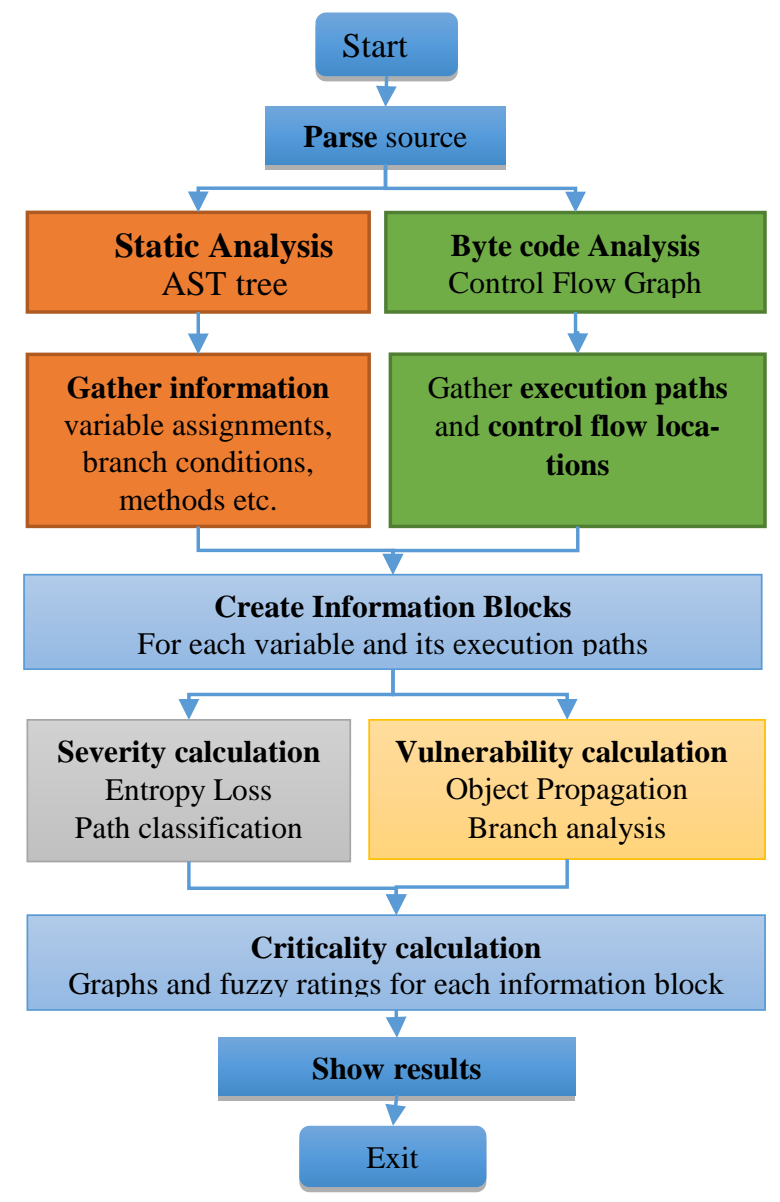

Figure 4: Entroine's processing flowchart.

\subsection{Taxonomy of dangerous instruc- tions}

Following Oracle's JAVA API and documentation (Java API Specification), (Gosling, 2013) and (Harold, 2006), three categories of Java instructions were 
used to classify execution paths in IBs. (i) Control Flow instructions, (ii) Input Vector instructions and (iii) potentially exploitable methods (sinks). About 159 Java methods were reviewed and gathered from formal publications and organizations that specifically classify exploits and, consequently, any instructions used in them (Mell, 2006) (Gosling, 2013) (Harold, 2006).

The taxonomy's methods were grouped into five categories of Severity corresponding to the taxonomy's Severity ranks. We based the Severity classification ranks for ranking instructions on the wellknown international Common Vulnerability Scoring System (CVSS) scoring system (Mell, 2006) and the Common Weakness Enumeration system (CWE CWSS). CVSS classifies potential vulnerabilities into danger levels and is used by NIST's National Vulnerability Database (NVD) to manage an entire database of vulnerabilities found in deployed software. The NVD is also using the CWE system as a classification mechanism that differentiates CVEs by the type of vulnerability they represent. The Common Weakness Scoring System (CWSS) provides a mechanism for prioritizing software weaknesses. It applies scores into vulnerabilities based on a mathematical formula of characteristics.

Entroine uses all three of these systems, NVDCVSS, CWE and CWSS ranking to assign source code instructions to specific danger levels, according the type of vulnerability, in which they participate, its general SWSS score and corresponding ranking value in similar CVSS vulnerabilities that we found.

Example: The Runtime.exec()instruction is widely-known to be used in many OS command injection exploits. CWE and NIST provide a multitude of critical vulnerabilities based in this instruction (e.g. the CWE-78 category). Also the CWSS 3.0 scoring system ranked the use of exec ( ) to execute code with application level privileges very high in its scale (9.3 out of 10). Thus, Entroine's taxonomy classifies the exec() instruction into its very high (5) danger level category. Similar notion has been followed in organizing the rest of Entroine's taxonomy instructions into Severity levels. This way, we limit our personal intuition, in an effort to support that Entroine's ranking system is justified.

Due to space limitations, only two small Java Class group examples are given. The complete classification system can be found in the provided link at the end of the article. The symbol $\S$ corresponds to chapters in Java documentation (Gosling, 2013):

\section{Control Flow Statements}

According to a report (NSA, 2011), boolean expressions determine the control flow. Such expressions are found in the following statements:
(1) if-statements (§14.9)
(2) switch-statements (\$14.11)
(3) while-statements (\$14.12)
(4) do-statements (\$14.13)
(5) for-statements (\$14.14)

\section{Input Vector Methods}

Java has numerous methods and classes that accept data from users, streams or files (Harold, 2006). Most of them concern byte, character and stream input/output. Entroine takes into account 69 different ways of entering data into an AUT. A small example is given below in Table 1 .

Table 1: Example group- Input Vector Methods taxonomy.

\begin{tabular}{|c|l|}
\hline java.io.BufferedReader & $\begin{array}{l}\text { java.io. } \\
\text { BufferedInputStream }\end{array}$ \\
\hline java.io.ByteArrayInputStream & $\begin{array}{l}\text { java.io. } \\
\text { DataInputStream }\end{array}$ \\
\hline java.lang.System & javax.servlet.http. \\
\hline java.io.ObjectInputStream & java.io.StringReader \\
\hline
\end{tabular}

Based on (Harold, 2006) and common programming experience, monitoring specific Java objects seems to be an adequate, albeit not entirely thorough, way of tracing user data inside Java applications.

\section{Exploitable Methods (sinks)}

Based on CWE, NVD (Mell, 2006) and common knowledge, we know that specific methods are used in exploits. We consider them as potential sinks and thus, Entroine examines them carefully. As mentioned earlier, Entroine's taxonomy of exploitable methods was based on the exploit classification and relevant source code by NIST's NVD in their CWE taxonomy (Mell, 2006). Entroine takes into account 90 methods known to be exploitable as sinks, according to NIST CWEs. It then classifies them according to CWE's rank and its corresponding CVSS (CWE) and CWSS rank. A small example is given below at Table 2:

Table 2: Example group - Sink methods taxonomy.

\begin{tabular}{|l|l|}
\hline java.lang.Runtime & java.net.URLClassLoader \\
\hline java.lang.System & java.sql.Statement \\
\hline $\begin{array}{l}\text { javax.servlet. } \\
\text { http.HttpServlet }\end{array}$ & javax.script \\
\hline java.io. File & java.net.Socket \\
\hline
\end{tabular}




\subsection{Classification and ranking system}

As explained in Section 3, the Fuzzy Logic system from (Cingolani, 2012) is used in Entroine, which provides a means to rank possible logical errors. In order to aid the end-user, Severity and Vulnerability values are grouped into 3 sets (Low, Medium, High), with an approximate width of each group of $5 / 3=$ $1,66 \sim 1,5$ (final ranges: Low in [0...2], Medium in $(2 \ldots 3,5]$ and High in $(3,5 \ldots 5])$.

\subsubsection{Calculating Severity using Entropy Loss and Feature Selection}

Entroine's classification system for execution path instructions and transitions uses Entropy Loss to capture the danger level in AUT's execution paths. It takes into consideration specific instruction appearances against the total number of instructions in a given set of transitions and applies Severity ranks to execution paths and the corresponding information blocks.

Entroine detects, evaluates and classifies instructions found in execution. Severity ratings are applied by classifying each information block into one of five Severity levels, according to the Prior Entropy and Entropy Loss of features in every execution path.

Since each information block refers to a specific execution path and its variables, the necessary metrics are calculated based on a ratio between path instructions considered dangerous (e.g. command execution instructions like exec( )) and the total number of instructions involved in the transitions of each path. Similarly to (Ugurel, 2002), probabilities for the expected entropy loss of each feature are calculated as follows:

$$
\begin{aligned}
& \operatorname{Pr}(C)=\frac{\text { numberOfPositiveInstructions }}{\text { totalNumberOf } \text { Instructions }} \\
& \operatorname{Pr}(\bar{C})=1-\operatorname{Pr}(C) \\
& \operatorname{Pr}(f)=\frac{\text { numberOfInstructionsWithFeatureF }}{\text { totalNumberOf Instructions }} \\
& \operatorname{Pr}(\bar{f})=1-\operatorname{Pr}(f) \\
& \operatorname{Pr}(C \mid f)= \\
& =\frac{\text { numberOfInstructionsWithFeatureF }}{\text { totalNumberOfInstructionsWithFeatureF }} \\
& \operatorname{Pr}(\bar{C} \mid f)=1-\operatorname{Pr}(C \mid f)
\end{aligned}
$$

$$
\begin{aligned}
& \operatorname{Pr}(C \mid \bar{f})= \\
& =\frac{\text { numberOfInstructionsWithoutFeatureF }}{\text { totalNumberOf InstructionsWithoutFeatureF }} \\
& \operatorname{Pr}(\bar{C} \mid \bar{f})=1-\operatorname{Pr}(C \mid \bar{f})
\end{aligned}
$$

numberOfPositiveInstructions denotes to the sum of instructions in a given path that belong to all danger levels regardless of category (low, medium or high), while numberOfInstructionsWithFeatureF represents the sum of instructions which belong to a specific danger level category (e.g. Severity rank 3), based on feature ranks.

Entropy Loss is computed separately for each source code feature characterized by a specific token. Only tokens that are part of a variable's execution paths are analyzed. For example, in the expression "data = properties.getproperty ("data");" the tokens will be: "data", "getProperty" and "properties".

The taxonomy of Java instructions in section 4.2 defines the various features used in place of $f$ in the above equations. An example of Entroine's features classification is given in Table 3 . For a complete list, the reader is referred to the link at the end of the article.

Table 3: Severity classification examples.

\begin{tabular}{|l|l|c|}
\hline Rank & $\begin{array}{l}\text { Example of classified meth- } \\
\text { ods }\end{array}$ & Category \\
\hline Low & javax.servlet.http.Cookie & 1 \\
\hline Low & java.lang.reflection.Field & 2 \\
\hline Medium & java.io.PipedInputStream & 3 \\
\hline High & java.io.FileInputStream & 4 \\
\hline High & java.sql.ResultSet::getString & 5 \\
\hline
\end{tabular}

\subsubsection{Calculating Vulnerability using Control Flow analysis and Tainted Object Propa- gation}

To calculate Vulnerability, Entroine runs a Tainted Propagation algorithm that classifies the likelihood of an exploit happening in an execution path. Entroine uses BCEL to traverse the program's Control Flow Graph bottom-to-top, in order to gather variable execution paths. Entroine's propagation rules are the following:

- The highest entropy loss (information gain) is detected Variables assigned data from expressions (e.g. +, -, method return) whose output depends on tainted data, are tainted.

- Literals (e.g. hardcoded strings, true declarations) are never tainted. 
- If an object's variable gets tainted, only data referred by that variable are considered tainted, not all object properties.

- Methods that accept tainted variables as parameters are considered tainted.

- The return value of a tainted function is always tainted, even for functions with implicit return statements (e.g. constructors).

Table 4 below depicts the check rules for exploit detection.

Table 4: Vulnerability check rules and their categories.

\begin{tabular}{|l|l|c|}
\hline Rank & $\begin{array}{l}\text { Example of classified } \\
\text { methods }\end{array}$ & Category \\
\hline Low & $\begin{array}{l}\text { No improper checks of vari- } \\
\text { ables. }\end{array}$ & 1 \\
\hline Low & $\begin{array}{l}\text { Sinks NOT linked to input } \\
\text { vectors. }\end{array}$ & 2 \\
\hline Medium & Propagation to methods & 3 \\
\hline High & $\begin{array}{l}\text { Improper checks on varia- } \\
\text { bles with input data - Varia- } \\
\text { bles used in sinks. }\end{array}$ & 4 \\
\hline High & $\begin{array}{l}\text { No checks - variables used in } \\
\text { sinks. }\end{array}$ & \multicolumn{2}{|c|}{5} \\
\hline
\end{tabular}

\subsubsection{Risk}

Risk represents a calculated value assigned to each information block IB and its corresponding variables, by aggregating the aforementioned Severity and Vulnerability ratings. Membership of an IB in Risk sets is calculated using Fuzzy Logic's IFTHEN rules (Fig. 2). For clarity, all scales (Severity, Vulnerability and Risk) are divided in the same sets: "Low", "Medium" and "High". Fig. 2 provides an example of how Risk is calculated using Fuzzy Logic linguistic rules:

\section{IF Severity=low AND Vulnerability=low THEN Risk=low}

Table 5 shows the fuzzy logic output for Risk, based on the aggregation of Severity and Vulnerability.

Table 5: Severity x Vulnerability = R - Risk sets.

\begin{tabular}{|c|c|c|c|}
\hline $\begin{array}{l}\text { Severity } \\
\text { Vulnerability }\end{array}$ & Low & Medium & High \\
\hline Low & Low & Low & Medium \\
\hline Medium & Low & Medium & High \\
\hline High & Medium & High & High \\
\hline
\end{tabular}

\section{EXPERIMENTS AND RESULTS}

\subsection{Entroine's architecture and work- flow}

In order to test our profiling approach implemented in Entroine, we needed appropriate AUTs to analyze. We considered whether we should use opensource software or "artificially made" programs, such as those usually used for benchmarking program analysis tools. Both options are characterized by various positive characteristics and limitations.

In choosing between real AUTs and artificial code for our purpose, we endorsed NSA's principles from (NSA, 2011)(NSA, 2012) were it states that "the benefits of using artificial code outweigh the associated disadvantages". Therefore, for preliminary experimentation with Entroine we have opted using the Juliet Test Case suite, a formal collection of artificially-made programs packed with exploits.

The Juliet Test Suite is a collection of over 81.000 synthetic $\mathrm{C} / \mathrm{C}++$ and Java programs with a priori known flaws. The suite's Java tests contain cases for 112 different CWEs (exploits). Each test case focuses on one type of flaw, but other flaws may randomly manifest. A bad( ) method in each test-program manifests an exploit. A good ( ) method implements a safe way of coding and has to be classified as a true negative. Since Juliet is a synthetic test suite, we mark results as true positive, if there is an appropriate warning in flawed (bad) code or false positive, if there is an appropriate warning in non-flawed (good) code, similarly to (Okun, 2013).

This testing methodology is developed by NIST. We focus on exploits from user input, whereas other categories are not examined (e.g. race conditions). Table 6 below provides a list of all Weakness Class Types used in the study. The middle column depicts the categories of exploits on which Entroine is tested (e.g. HTTP Response/Req Header-Servlet (add): exploits that manifest on servlets when adding HTTP headers in responses and requests):

Table 6: Weakness Classes - CWE.

\begin{tabular}{|l|l|l|}
\hline $\begin{array}{l}\text { Weakness - } \\
\text { CWE }\end{array}$ & $\begin{array}{l}\text { Types of Weaknesses } \\
\text { analyzed }\end{array}$ & $\begin{array}{l}\text { Num } \\
\text { of } \\
\text { Tests }\end{array}$ \\
\hline \hline & $\begin{array}{l}\text { HTTP Response/Req Header- } \\
\text { Servlet (add) }\end{array}$ & 15 \\
CWE-113 & $\begin{array}{l}\text { HTTP Response/Req Cookie } \\
\text { Servlet } \\
\text { HTTP Response/Req Header- } \\
\text { Servlet (set) }\end{array}$ & \\
\hline
\end{tabular}




\begin{tabular}{||l|l|c|}
\hline CWE-78 & $\begin{array}{l}\text { Operating System Com- } \\
\text { mand_Injection }\end{array}$ & 15 \\
\hline CWE-89 & $\begin{array}{l}\text { SQL Injection_connect_tcp } \\
\text { SQL Injec- } \\
\text { tion_Environment_execute } \\
\text { SQL Injec- } \\
\text { tion_Servlet_execute }\end{array}$ & 15 \\
\hline
\end{tabular}

We ran Entroine on a set of vulnerable sample programs from the CWE categories depicted in Table 6 . Our test data set consists of 45 total Juliet programs, 15 cases from each CWE category depicted in Table 6. Each bad method with an exploit will have to produce a True Positive (TP), whereas all good methods will have to represent True Negatives (TN). Overall 178 tests $(\mathrm{TP}+\mathrm{TN})$ were included in all programs: 45 exploits and 133 cases of safe implementations (TNs). Entroine flags detections when both Severity and Vulnerability ranks for an IB are ranked above the Low zone (Risk $>=3$ ). Table 7 shows the overall results of our tests and, consequently, the accuracy of the tool:

Table 7: TP, TN, FP detection rate and accuracy in 80 samples.

\begin{tabular}{|l|l|l|l|l|l|}
\hline $\begin{array}{l}\text { Weakness } \\
\text { Class - } \\
\text { CWE }\end{array}$ & $\begin{array}{l}\text { TP } \\
\text { Rate }\end{array}$ & $\begin{array}{l}\text { TN } \\
\text { Rate }\end{array}$ & $\begin{array}{l}\text { TP+ } \\
\text { TN }\end{array}$ & $\begin{array}{l}\text { All } \\
\text { tests }\end{array}$ & $\begin{array}{l}\text { Num of } \\
\text { pro- } \\
\text { grams }\end{array}$ \\
\hline $\begin{array}{l}\text { CWE sam- } \\
\text { ples }\end{array}$ & $\begin{array}{l}45 / \\
45\end{array}$ & $\begin{array}{l}133 / 1 \\
33\end{array}$ & 178 & 178 & $\begin{array}{l}45 \\
\text { pro- } \\
\text { grams }\end{array}$ \\
\hline $\begin{array}{l}\text { ACCU- } \\
\text { RACY }\end{array}$ & \multicolumn{3}{|c|}{ TP $=100 \%, \mathrm{FP}=0 \%$} \\
\hline
\end{tabular}

Table 8 provides a more detailed view of the results shown in Table 7. Table 8 depicts all tests per category of Juliet programs whereas Table 7 is an overall look on the results. 15 differentiated tests from each category where chosen for Entroine's preliminary proof-of-concept:

Table 8: Detection rates for each Weakness Type.

\begin{tabular}{|l|l|l|l|l|l|}
\hline $\begin{array}{l}\text { Weakness } \\
\text { Class - CWE }\end{array}$ & TP & TN & $\begin{array}{l}\text { TP+ } \\
\text { TN }\end{array}$ & $\begin{array}{l}\text { All } \\
\text { tests }\end{array}$ & $\begin{array}{l}\text { Num of } \\
\text { pro- } \\
\text { grams }\end{array}$ \\
\hline $\begin{array}{l}\text { CWE-89: SQL } \\
\text { Injection }\end{array}$ & $\begin{array}{l}15 / 1 \\
5\end{array}$ & $51 / 51$ & 66 & 66 & $\begin{array}{l}15 \text { pro- } \\
\text { grams }\end{array}$ \\
\hline $\begin{array}{l}\text { CWE-78: OS } \\
\text { Command } \\
\text { Injection }\end{array}$ & $\begin{array}{l}15 / 1 \\
5\end{array}$ & $28 / 28$ & 43 & 43 & $\begin{array}{l}15 \text { pro- } \\
\text { grams }\end{array}$ \\
\hline $\begin{array}{l}\text { CWE-113: } \\
\begin{array}{l}\text { HTTP Re- } \\
\text { sponse Split }\end{array}\end{array}$ & 5 & $54 / 54$ & 69 & 69 & $\begin{array}{l}15 \text { pro- } \\
\text { grams }\end{array}$ \\
\hline
\end{tabular}

\section{CONCLUSIONS}

It should be noted that Entroine is in pre-alpha stage. Tests act as proof-of-concept statistics since testing real-world, big applications is not yet feasible due to package complexity, external libraries etc. State explosion remains an issue, a problem inherited by the used analysis techniques. Yet, state explosion seems manageable using source code classification to focus on specific variable paths. Severity ranking helps this.

Also, a limitation of Entroine is that it cannot detect errors based on variables' context. This needs semantic constructs to analyze information behind input data. A formal comparison with known tools is needed.

We plan on using this technique to test real-world code used in cyber-physical systems (e.g. high level code that manipulates devices through SCADA systems). This will work as an extension to previous work of ours in the area (Stergiopoulos, 2015).

Entroine runs relatively fast in comparison to what it has to do. Table 9 depicts execution times.

Table 9: Entroine's execution times.

\begin{tabular}{|l|l|}
\hline $\begin{array}{l}\text { Execution time - per 15 } \\
\text { tests: }\end{array}$ & $129 \mathrm{sec}(\sim 2 \mathrm{~min})$ \\
\hline $\begin{array}{l}\text { Entropy Loss calculation } \\
\text { (per test) }\end{array}$ & 1 millisecond \\
\hline Static analysis (per test) & $\sim 5 \mathrm{sec}$ \\
\hline
\end{tabular}

All tests were ran on an Intel Core i5 4570 PC (3.20 GHz, 8GB RAM). An anonymous link to Entroine's taxonomy and example files is given below.

ht tps://dl.dropboxusercontent . com/u /48299252/Entroine_files.zip

\section{REFERENCES}

Boland T., Black P., 2012. Juliet 1.1 C/C++ and Java Test Suite”. In Computer, vol. 45, no. 10, pp. 88-90.

The National Security Agency (NSA) Center for Assured Software (CAS), Analyzing Static Analysis Tools, 2011.

Rutar, N., Almazan, C. B. and Foster, S. J., 2004. A Comparison of Bug Finding Tools for Java. In Proceedings of the 15th International Symposium on Software Reliability Engineering (ISSRE '04). IEEE Computer Society, Washington, DC, USA, 245-256.

Livshits V. and Lam M., 2005. Finding security vulnerabilities in Java applications with static analysis. In Proceedings of the 14th Usenix Security Symposium. 
Ayewah, N. Hovemeyer, D. Morgenthaler, J.D. Penix, J. and Pugh, W., 2008. Using Static Analysis to Find Bugs. In Software, IEEE, vol.25, no.5, pp.22,29.

CodePro, https://developers.google.com/java-devtools/codepro/doc/

UCDetector, http://www.ucdetector.org/ Pmd, http://pmd.sourceforge.net/

Tripathi, A. K. and Gupta, A. 2014. A controlled experiment to evaluate the effectiveness and the efficiency of four static program analysis tools for Java programs. In Proceedings of the 18th International Conference on Evaluation and Assessment in Software Engineering (EASE '14). ACM, New York, NY, USA

Hovemeyer, D. and Pugh, W. 2004. Finding bugs is easy. In SIGPLAN Not. 39, 12, pp. 92-106.

Jovanovic, N., Kruegel, C. and Kirda E. 2010. Static analysis for detecting taint-style vulnerabilities in web applications. In Journal of Computer Security, Issue 5, IOS Press,

Weiser M., 1981. Program Slicing. In Proc. of the International Conference on Software Engineering, pp. 439449 ,

Stergiopoulos, G., Tsoumas, V. and Gritzalis, D., 2013. On Business Logic Vulnerabilities Hunting: The APP_LogGIC Framework. In Proc. of the $7^{\text {th }}$ Int. Conf. on Network and System Security. Springer, 236249.

Zhang X., Gupta N., Gupta R. 2006. Pruning Dynamic Slices with Confidence. In Proc. of the Conference on Programming Language Design and Implementation, pp. 169-180.

Cingolani P., Alcala-Fdez J., 2012. jFuzzyLogic: A robust and flexible Fuzzy-Logic inference system language implementation”. In IEEE International Conference on Fuzzy Systems, 1-8.

Doupe A., Boe B. Vigna G. 2011. Fear the EAR: Discovering and Mitigating Execution after Redirect Vulnerabilities. In Proc. of the 18th ACM Conference on Computer and Communications Security. ACM, USA, pp. 251-262.

Balzarotti D., Cova M., Felmetsger V., Vigna G., 2007. Multi-module vulnerability analysis of web-based applications. In: Proc. of the 14th ACM Conference on Computer and Communications security. ACM, USA, 25-35.

Albaum G., “The Likert scale revisited”. In Market Research Society Journal, vol. 39, pp. 331-348, 1997.

Ugurel, S., Krovetz, R., Giles, C. L., Pennock, D. M., Glover, E. J. Zha, H. 2002. What's the code?: automatic classification of source code archives. In Proc.of the 8th ACM SIGKDD international conference on Knowledge discovery and data mining (ACM KDD '02), ACM, USA pp. 632-638.

Abramson, N. "Information Theory and Coding." McGraw-Hill, New York, 1963.

Etzkorn, L. and Davis, C. G. 1997. Automatically identifying reusable OO legacy code. In IEEE Computer, pp. 66-71.
Glover, E. J., Flake, G. W., Lawrence, S., Birmingham, W. P., Kruger, A., Giles, L. C., and Pennoek, D. M. 2001. Improving category specific web search by learning query modification. In IEEE Symposium on Applications and the Internet (SAINT 2001), IEEE press, USA, pp. 23-31.

Stoneburner G. and Goguen A. 2002. SP 800-30. Risk Management Guide for Information Technology Systems. Technical Report. NIST MD, USA.

The OWASP Risk Rating Methodology, www.owasp.org/ index.php/OWASP_Risk_Rating_Methodology.

Leekwijck W., Kerre E., 1999. Defuzzification: Criteria and classification. In Fuzzy Sets and Systems, vol. 108, issue 2, 159-178.

Java Platform, Standard Edition 7 API Specification, http://docs.oracle.com/javase/7/docs/api/

Gosling J., Joy B., Steele G., Bracha G., Buckley A., The Java Language Specification, Java SE 8 Edition, 2013 http://docs.oracle.com/javase/specs/jls/se8/html/index. html

Harold E., 2006. Java I/O, Tips and Techniques for Putting I/O to Work. O’Reilly.

On Analyzing Static Analysis Tools. 2011. Center for Assured Software, National Security Agency.

Static Analysis Tool Study-Methodology. 2012. Center for Assured Software, National Security Agency.

Yang, Y. and Pederson, J. 1997. A comparative study on feature selection in text categorization. In Proceedings of the Fourteenth International Conference on $\mathrm{Ma}$ chine Learning (ICML'97), 412-420

Apache Commons BCEL project page. http://commons.apache.org/proper/commons-bcel/

Okun, V., Delaitre, O. and Black, P. E., 2013. Report on the Static Analysis Tool Exposition (SATE) IV, NIST Special Publication 500-297.

Stergiopoulos, G., Tsoumas, B. and Gritzalis, D., 2012. Hunting application-level logical errors. In Proceedings of the Engineering Secure Software and Systems Conference (ESSOS '12). Springer (LNCS 7159), 135142.

Stergiopoulos G., Katsaros P., Gritzalis D., 2014. Automated detection of logical errors in programs". In: Proc. of the 9th International Conference on Risks and Security of Internet and Systems (CRiSIS-2014), Springer

Coverity SAVE audit tool, http://www.coverity.com.।

Mell, P.; Scarfone, K.; Romanosky, S., 2006. Common Vulnerability Scoring System. In Security \& Privacy, IEEE, vol.4, no.6, pp.85-89.

The Common Weakness Enumeration (CWE), Office of Cybersecurity and Communications, U.S. Department of Homeland Security, http://cwe.mitre.org

Stergiopoulos G., Theoharidou M., Gritzalis D., 2015. Using logical error detection in Remote-Terminal Units to predict initiating events of Critical Infrastructures failures. In Proc. of the 3rd International Conference on Human Aspects of Information Security, Privacy and Trust (HCI-2015), Springer, USA. 